\section{EDITORIAL}

EDITORIAL

\title{
A crescente vitalidade das produções científicas sobre o envelhecimento
}

The growing vitality of scientific productions on

aging

La creciente vitalidad de las producciones científicas sobre el envejecimiento

Flamínia Manzano Moreira Lodovici Elisabeth Frohlich Mercadante

Estimados leitores-amigos que nos honram com sua presença aqui. Sejam todos bem-vindos a este volume da Revista Kairós - Gerontologia, filiada à Faculdade de Ciências Humanas e da Saúde (FACHS), da Pontifícia Universidade Católica de São Paulo (PUC-SP). Com grande satisfação apresentamos o volume 20(2), desta revista, com um total de 25 trabalhos em 2 modalidades: 22 artigos científicos, 3 relatos de experiência, ainda mais que, tributariamente aos esforços da Reitoria da PUC-SP, todos estão identificados com seu DOI.

O título deste Editorial tenta dizer do vigor, do entusiasmo, com que vêm sendo produzidos escritos sobre o envelhecimento, submetidos para publicação neste periódico, constituindo cada um o resultado de pesquisas, reflexões, experiências, levadas a efeito por pesquisadores de diversas áreas e universidades de várias regiões do Brasil e do exterior. Os trabalhos recebidos por esta revista são gerados, via de regra, por Universidades Federais e Estaduais, mas o que nos vem surpreendendo é que, cada vez mais, nos cheguem artigos de universidades particulares, algumas vocacionais ou de âmbito religioso, o que nos evidencia o quanto a emergente problemática do envelhecimento está sendo pesquisada nesse âmbito. Salta-nos aos olhos a formação eclética que hoje se observa dos autores: a graduação em uma área do conhecimento, o mestrado, o doutorado, o pós-doutorado em outras áreas que se interfaciam com a primeira em termos de concentração em uma temática similar de pesquisa voltada à problemática do envelhecimento. Seriamos a seguir os 25 trabalhos deste volume 20(2):

Um $\mathbf{1}^{\mathbf{0}}$ artigo recebido de Lisboa (Portugal), escrito por $\mathbf{2}$ pesquisadoras, enfermeiras da Escola Superior de Saúde Dr. Lopes Dias, Instituto Politécnico de Castelo Branco, identifica-se com o doi: http://dx.doi.org/10.23925/2176901X.2017v20i2p9-26. 
Sob o título "Construção Social do Envelhecimento Individual”, apresenta-se reflexão acerca do envelhecimento individual, realizada durante a investigação acerca da Promoção e Preservação da Dignidade no contexto de cuidados em lares de idosos.

Um $\mathbf{2}^{\mathbf{0}}$ artigo submetido em inglês, vindo de Goiás, escrito por $\mathbf{2}$ pesquisadores, sendo o primeiro, pesquisador e docente de literatura, ligado ao Programa de PósGraduação em Letras e Linguística da Universidade Federal de Goiás, UFG; o segundo, administrador de empresas, ligado à Fundação Getúlio Vargas, FGV/SP. Sob o título "Crazy Lady is an early old woman and needs care", e a identificação doi: http://dx.doi.org/10.23925/2176-901X.2017v20i2p27-44, os autores elaboraram uma reflexão que faz interface entre literatura e envelhecimento, iniciando com uma citação: "A velhice é uma forma de sentir frio que me assaltal e uma certa acidez": o começo de um poema de Adélia Prado, no qual ela trata da temática do envelhecimento, da velhice e do cuidado que é necessária nessa fase da vida, a preocupação com a pessoa idosa, as práticas instrumentais de cuidado, as condições biopolíticas humanitárias e democráticas que podem otimizar a vida de idosos em nossa sociedade.

O $3^{\text {o }}$ artigo vindo da Bahia, de título "Condições de saúde psicológica, capacidade funcional e suporte social de idosos", foi submetido a este periódico por 2 pesquisadoras, ambas psicólogas, e filiadas à Universidade Federal do Recôncavo da Bahia. Este estudo que recebeu o doi: http://dx.doi.org/10.23925/2176901X.2017v20i2p45-58, avaliou sintomas depressivos e de ansiedade em idosos, e sua associação com o sexo, a faixa etária, a capacidade funcional e a percepção de suporte social. Pontua-se que é importante maior atenção pública para o diagnóstico e tratamento dessas condições clínicas.

O $4^{\circ}$ artigo chegou de Brasília, DF, de assistente social ligada à Universidade de Brasília, UnB, registrado como: http://dx.doi.org/10.23925/2176901X.2017v20i2p59-78. Sob o título "Dependência e Pessoas idosas: significado e políticas", o presente artigo aborda aspectos sociodemográficos referentes ao envelhecimento populacional e ao aumento da expectativa de vida, destacando-se a população com 80 anos e mais de idade. Para esse grupo, a dependência é reconhecida como um risco social, que demanda cuidados de longa duração, tema que vem sendo discutido há mais de duas décadas nos países desenvolvidos.

De Portugal, chegou o $\mathbf{5}^{\mathbf{0}}$ artigo deste volume 20(2), de $\mathbf{1}$ pesquisador das Ciências Sociais, Especialidade de Administração da Saúde, filiado ao Instituto Superior de Ciências Sociais e Políticas (ISCSP), da Universidade de Lisboa. Este estudo, de título "Modernização, envelhecimento e infoexclusão em Portugal", registrado como http://dx.doi.org/10.23925/2176-901X.2017v20i2p79-99, considera que o grande desenvolvimento das tecnologias de informação geraram, em Portugal, uma maior dependência para tratar de assuntos quotidianos, como o acesso aos serviços bancários, finanças, marcação de consultas, levantamento de medicamento nas farmácias e até compras. 
O $6^{\circ}$ artigo recebido de pesquisadores 2 Estados brasileiros: Santa Catarina e Rio Grande do Sul, sendo 5 pesquisadoras: 2 educadoras físicas, da Universidade Federal de Santa Catarina, UFSC, Florianópolis, SC; 1 fisioterapeuta, da Faculdade de Educação Física e Fisioterapia da Universidade de Passo Fundo, RS; 2 graduadas em Envelhecimento humano, do Programa de Pós-Graduação em Envelhecimento Humano, Universidade de Passo Fundo, RS. Registrado como: doi: http://dx.doi.org/10.23925/2176-901X.2017v20i2p101-116, o título é: "Associação entre número de quedas e força muscular de idosos residentes em instituições de longa permanência". Observou-se que o programa de exercícios físicos proposto contribuiu na melhora da força muscular e, consequentemente, na melhora do risco de quedas.

O $\mathbf{7}^{\mathbf{0}}$ artigo de São Paulo, vindo de 2 psicólogas da PUC-SP, registrado como doi: http://dx.doi.org/10.23925/2176-901X.2017v20i2p117-133, sob o título "Reflexões acerca do envelhecimento, problemáticas, e cuidados com as pessoas idosas", objetiva apresentar considerações teóricas acerca da vivência e enfrentamento do envelhecimento, enquanto processo e representação, associado ao adoecimento e à necessidade de cuidados familiares e/ou especializados.

O $\mathbf{8}^{\mathbf{0}}$ artigo advindo de Brasília, tem $\mathbf{3}$ pesquisadoras, sendo $\mathbf{2}$ enfermeiras e $\mathbf{1}$ fisioterapeuta: a primeira atua como Chefe do Centro Cirúrgico do Hospital das Forças Armadas, Brasília, DF. A segunda, ligada ao Programa de Mestrado Profissional em Ciências para a Saúde, ESCS/FEPECS/DF, Brasil. A terceira é Doutora em Investigação Biomédica, Departamento de Clínica Médica, Divisão de Geriatria, Faculdade de Medicina de Ribeirão Preto da Universidade de São Paulo, MRP/USP, e Professora do Programa de Pós-Graduação Stricto Sensu em Gerontologia, Universidade Católica de Brasília, UCB. O estudo registrado como doi: http://dx.doi.org/10.23925/2176-901X.2017v20i2p135-160, e intitulado "Síndrome da Paralisia Supranuclear Progressiva: Há dificuldades de diagnóstico e a atuação da Equipe multidisciplinar quanto aos cuidados à pessoa afetada por esta doença. $\mathrm{O}$ objetivo do estudo foi investigar a fisiopatologia, diagnóstico, tratamento e assistência da equipe multidisciplinar às pessoas com PSP. O estudo revelou pouca publicação e, por ser rara, não existe fármaco eficiente e eficaz; o diagnóstico é limitado nas primeiras manifestações, e somente possível por meio de exames mecanicistas. Sugere-se que os Conselhos, Associações de Neurologia e demais especialidades envolvidas no seu tratamento divulguem mais detalhes sobre a doença, a fim de se criar um protocolo de atendimento integral aos afetados pela síndrome, bem como o necessário apoio aos familiares e cuidadores, que auxilie nas práticas da assistência ambulatorial e familiar.

O $9^{\circ}$ artigo vindo do estado do Paraná, é de $\mathbf{2}$ pesquisadores: 1 médico e $\mathbf{1}$ fisioterapeuta, do Serviço de Neurologia, Hospital de Clínicas da Universidade Federal do Paraná, HC/UFPR. Registrado sob o doi: http://dx.doi.org/10.23925/2176901X.2017v20i2p161-178, de título "Fatores associados ao desempenho funcional de idosos portadores da Doença de Parkinson”. 
Seu objetivo foi identificar fatores associados ao perfil funcional de idosos com essa doença, para correlacioná-los com a gravidade da doença e o tempo de diagnóstico em três grupos etários. A DP está acometendo cada vez mais a população, e fatores como baixa escolaridade e viuvez foram relacionados a déficits funcionais, levando-se à questão da fragilidade física e potenciais fatores para quedas. O grupo G3 (80-89 anos) foi o mais afetado funcionalmente e o tempo de diagnóstico não foi relacionado com a idade. $\mathrm{O} \mathbf{1 0}^{\circ}$ artigo recebido da capital paulistana, de $\mathbf{2}$ pesquisadoras: uma arquiteta e a outra psicóloga, ambas filiadas a Cursos de Graduação e Pós-Graduação em Gerontologia, da Universidade de São Paulo (USP), Escola de Artes, Ciências e Humanidades (EACH). Registrado sob o doi: http://dx.doi.org/10.23925/2176-901X.2017v20i2p179-194 e com título: "O espaço urbano do bairro e o impacto nas relações sociais de idosos: uma revisão narrativa da literatura", o estudo objetivou apresentar a relação entre o meio urbano e as relações sociais na velhice. Demonstrou-se que as condições ambientais do bairro podem influenciar as relações sociais de seus moradores idosos, intensificando o isolamento dos sujeitos quando carece de recursos, e promovendo a socialização, com oportunidades para o caminhar e a troca social.

O $\mathbf{1 1}^{\mathbf{0}}$ artigo recebido de Marília, de $\mathbf{3}$ pesquisadores: uma terapeuta ocupacional ligada à UNESP-Rio Claro; um segundo, fisioterapeuta; e um terceiro, neurocientista, ambos ligados à UNESP Marília, SP. Com doi: http://dx.doi.org/10.23925/2176-901X.2017v20i2p195-210, e título "Depressão, Declínio Cognitivo e Polimedicação em idosos institucionalizados", a pesquisa objetivou analisar possíveis relações entre depressão, declínio cognitivo e consumo de medicamentos. Em função da polimedicação, os sujeitos se enquadram nos fatores de risco para 'problemas relacionados ao uso de medicamentos' (PRM's). Foram observadas correlações entre os indicativos de depressão e declínio cognitivo. Não foram observadas correlações entre as demais variáveis analisadas.

O $12^{\circ}$ artigo recebido da capital paulistana, de $\mathbf{3}$ pesquisadores, sendo 2 psicólogas, uma terapeuta em consultório e outra da PUC-SP, e 1 da Tecnologia da Computação. Sob o doi: http://dx.doi.org/10.23925/2176-901X.2017v20i2p211-228 e título: "Avaliação dos aspectos da Personalidade em pessoas idosas na cidade de São Paulo por meio do Rorschach Performance System (R-PAS)", o artigo objetivou identificar aspectos da personalidade que predominam em uma amostra de idosos. Foi possível verificar uma visão mais simplista e distorcida da realidade, embora sem confusão de pensamento, além de dificuldade cognitiva e necessidade maior de estimulação diante das demandas do ambiente.

O $\mathbf{1 3}^{\mathbf{0}}$ artigo recebido do Rio Grande do Sul, de 4 pesquisadores: 1 psicólogo, bacharel em Direito, ligado à Gerontologia Biomédica, do Instituto de Geriatria e Gerontologia (IGG), na Pontifícia Universidade Católica do Rio Grande do Sul (PUCRS); 1 advogada. Doutora em Gerontologia Biomédica e docente da Faculdade de Direito da UNICNEC, Osório; 2 médicos-psiquiatras. 
Ligados ao Programa de Pós-Graduação em Gerontologia Biomédica, da Pontifícia Universidade Católica do Rio Grande do Sul (PUCRS). Com doi: http://dx.doi.org/10.23925/2176-901X.2017v20i2p229-245, e de título "Revisão integrativa sobre os direitos trabalhistas dos cuidadores de idosos", o presente estudo sugere que haja mais atenção com os direitos trabalhistas, a fim de tornar o cuidado efetivo tanto para quem cuida, quanto para quem é cuidado.

O $14^{\mathbf{0}}$ artigo, vindo de Botucatu (SP) de 2 pesquisadoras: a primeira, gerontóloga e Doutora em Avaliação em Saúde; a segunda, médica sanitarista, filiada ao Departamento de Saúde Coletiva; ambas da Faculdade de Medicina de Botucatu (SP). Universidade Estadual Paulista Júlio de Mesquita Filho. Sob o registro http://dx.doi.org/10.23925/2176-901X.2017v20i2p247-269, e título "Atenção à saúde da pessoa idosa e ao envelhecimento em uma Rede de Serviços de Atenção Primária", o estudo objetivou analisar os serviços de Atenção Primária à Saúde quanto à oferta e organização de ações ao envelhecimento e à saúde da pessoa idosa, de uma Rede Regional de Atenção à Saúde do centro-oeste paulista. Os resultados sugerem que estes serviços desenvolvem ações de atenção ao envelhecimento de modo incipiente e limitado às doenças crônicas não transmissíveis.

O $\mathbf{1 5}^{\circ}$ artigo vindo da capital paulistana, foi recebido de $\mathbf{3}$ pesquisadores: uma primeira, Bacharel em Gerontologia e mestranda em Gerontologia, da Universidade de São Paulo (USP). Os dois seguintes, psicólogos, com atuação na área da Gerontologia da USP-SP. Com doi: http://dx.doi.org/10.23925/2176901X.2017v20i2p271-292, e título "Amor, relacionamentos amorosos e poliamor na perspectiva de jovens universitários e idosos", objetivou investigar as crenças de jovens universitários e idosos sobre: os conceitos por eles atribuídos à palavra amor, e o que entendem como relacionamentos amorosos, sejam estes monogâmicos, poligâmicos ou poliamorosos. Observou-se que os conceitos de amor, relacionamentos amorosos, e poliamor são heterogêneos, sendo influenciados pela vivência de cada indivíduo. Alguns fatores externos podem ter contribuído para a formação da concepção do indivíduo, como a cultura, o grupo social, a época vivida etc.

O $\mathbf{1 6}^{\mathbf{0}}$ artigo enviado de Vitória da Conquista, Bahia, por 3 pesquisadoras ligadas à Universidade Estadual do Sudoeste da Bahia, UESB, sendo: 1 enfermeira e 2 fisioterapeutas, sob o doi: http://dx.doi.org/10.23925/2176-901X.2017v20i2p293$\underline{309}$, e título "Repercussões psicossociais da dependência funcional no cotidiano de idosos longevos". O artigo objetiva discutir as repercussões psicossociais da dependência funcional no cotidiano de sete idosos longevos de uma Unidade de Saúde da Família. Ficou evidente, entre as repercussões psicológicas, a presença de sentimentos de medo, tristeza e depressão. Em relação a repercussões sociais, a principal encontrada foi a redução nas relações sociais e o isolamento social, com consequente solidão. 
O $\mathbf{1 7}^{\mathbf{0}}$ artigo recebido da cidade de Salvador (estado da Bahia), de $\mathbf{2}$ pesquisadoras ligadas à Universidade Católica do Salvador (UCSal), sendo 1 pedagoga, ligada ao curso de Graduação e Pós-Graduação da Faculdade Batista Brasileira (FBB); a outra é psicóloga clínica. Sob o registro do doi: http://dx.doi.org/10.23925/2176-901X.2017v20i2p311-332, e com o título "O avô e a avó na visão dos netos", este artigo tem o objetivo de descrever a visão dos netos sobre as relações com seu avô e sua avó, com base na dimensão pessoa da teoria bioecológica, de Bronfenbrenner. Os principais resultados apontam que há diferença nas relações intergeracionais em função de gênero.

O $\mathbf{1 8}^{\mathbf{0}}$ artigo foi recebido de Fortaleza (Ceará), de $\mathbf{3}$ pesquisadoras, todas psicólogas, filiadas ao Centro Universitário Estácio do Ceará. Sob o doi: http://dx.doi.org/10.23925/2176-901X.2017v20i2p333-352, intitulado: "Reflexões acerca dos Impactos Psicossociais da Institucionalização de Idosos no Brasil", o artigo objetiva discutir os impactos psicossociais da institucionalização de idosos no Brasil. Observou-se que os impactos psicossociais estão relacionados à exclusão, ao sofrimento psíquico, à falta de autonomia e de planos futuros, aos sentimentos de abandono, patologias, distúrbios do sono e uso de psicofármacos.

O $\mathbf{1 9}^{\mathbf{0}}$ artigo vindo do Rio de Janeiro (RJ), recebido de $\mathbf{3}$ pesquisadoras, todas atuando na área da psicologia: 1 primeira, psicóloga; uma segunda, mestra em psicologia, graduada em Letras; uma terceira, educadora física, bacharel em Serviço Social, com atuação em psicologia. Sob o doi: http://dx.doi.org/10.23925/2176901X.2017v20i2p353-374 e o título "Envelhecimento, autocuidado e memória: intervenção como estratégia de prevenção", o artigo objetiva analisar a relação entre envelhecimento, autocuidado e memória; apresentar as pesquisas sobre autocuidado no Brasil e os profissionais que trabalham com este conceito. Verifica-se que os idosos relatam dificuldade de guardar e recuperar informações, o que interfere no autocuidado. Exige-se participação ativa, responsável, influi na autonomia e independência, favorecendo a integração do idoso na família/comunidade. Os autores ressaltam que a intervenção é eficaz e benéfica para desenvolver o autocuidado.

O $\mathbf{2 0}^{\mathbf{0}}$ trabalho, advindo de Minas Gerais, de $\mathbf{5}$ pesquisadores todos ligados à Faculdade de Ciências Médicas de Minas Gerais. Sob o doi: http://dx.doi.org/10.23925/2176-901X.2017v20i2p375-398 e título "Estilo de vida e intervenções não farmacológicas no tratamento e na prevenção das síndromes geriátricas: uma revisão integrativa", considera-se que as grandes síndromes geriátricas - insuficiência cognitiva, instabilidade postural, incontinência urinária, iatrogenia e imobilidade - associam-se ao declínio da capacidade funcional de importante parcela idosa. Este trabalho faz uma compilação e análise de estudos que abordam a importância do estilo de vida, da alimentação, de exercícios físicos, e de outras medidas não farmacológicas, na prevenção e no tratamento dos chamados "gigantes da geriatria", condições de alta prevalência e morbidade na população idosa. 
O $21^{\circ}$ artigo foi recebido de Guarapuava (PR), de 7 pesquisadores, sendo 2 fisioterapeutas e 5 educadores físicos, filiados ao Programa de Pós-Graduação Stricto Sensu em Desenvolvimento Comunitário da Universidade Estadual do Centro-Oeste, Unicentro. Sob o doi: http://dx.doi.org/10.23925/2176-901X.2017v20i2p399-411, e título: "Comparação das pressões respiratórias máximas em idosos que praticam exercícios no solo e água", o artigo pretendeu comparar a força muscular respiratória em idosos saudáveis praticantes de exercícios em dois meios, solo e água. Os resultados demonstraram não haver alterações significativas na musculatura respiratória de ambos os grupos.

O $\mathbf{2 2}^{\circ}$ artigo advindo de Rio Claro (SP), de 7 pesquisadores, sendo 3 educadores físicos, 1 gerontóloga, 1 das Ciências Matemáticas e de Computação, ICMC, Universidade de São Paulo, 1 é médico veterinário, da Universidade de Buenos Aires. atualmente filiado ao Depart. de Ciências Exatas da ESALQ/USP, 1 médico da Universidade Estadual Paulista, UNESP, Departamento de Educação Física, câmpus de Rio Claro. Rio Claro, SP, Brasil. Sob o doi: http://dx.doi.org/10.23925/2176901X.2017v20i2p413-429, e título "Impacto das principais barreiras percebidas à prática de atividade física por aposentados de um município paulista”, este estudo avaliou o nível de atividade física, índice de massa corporal e as principais barreiras percebidas que impedem ou dificultam a prática de atividade física (tempo livre suficiente; realiza atividade física suficiente; falta de companhia; falta de dinheiro, dentre outras), bem como conhecer o perfil sociodemográfico dessa população, a fim de reconhecer possíveis deficiências e propor novas estratégias que possam subsidiar a melhora na sua qualidade de vida. Assim, torna-se importante ressaltar esses aspectos com relação à prática regular de atividade física, cujas barreiras podem ser transpostas se os profissionais e formuladores de políticas públicas implantarem programas voltados para esta população que visem ao incentivo dessa prática.

O $\mathbf{2 3}^{\circ}$ trabalho - um Relato de Experiência - advindo da capital paulistana, enviado por 1 pesquisadora assistente social, com mestrado em Gerontologia, graduação em Pedagogia. Sob o doi: http://dx.doi.org/10.23925/2176901X.2017v20i2p431-446 e título "O agir solidário de mais de vinte mil líderes comunitários em todo o território nacional em favor de um envelhecer mais saudável: relatos da experiência da Pastoral da Pessoa Idosa", o trabalho traz o relato de experiência da Pastoral da Pessoa Idosa, em que se cultua a experiência bem-sucedida de mais de vinte mil líderes comunitários no Brasil, que desempenham seu trabalho voluntariamente, em favor de um envelhecer mais saudável.

O 24 ${ }^{\circ}$ trabalho - outro Relato de Experiência - advindo de Santa Maria, RS, Brasil, recebido de 3 pesquisadoras, terapeutas ocupacionais, ligadas à Universidade Federal de Santa Maria. Santa Maria, RS, Brasil. Sob o doi: http://dx.doi.org/10.23925/2176-901X.2017v20i2p447-459, e título "Terapia Ocupacional no contexto institucional: um relato de experiência", o trabalho objetiva a experienciação dos discentes na compreensão do vínculo e do contexto institucional. 
Esse trabalho reafirma a importância do vínculo entre profissional e paciente, para que a aderência às propostas terapêuticas tenha maior compreensão e acolhimento, atuando como facilitadores da funcionalidade do cotidiano dos pacientes.

O $\mathbf{2 5}^{\circ}$ trabalho - um Relato de Experiência - recebido de Três Lagoas (MS), de uma enfermeira, pesquisadora ligada à Universidade Federal de Mato Grosso do Sul. Três Lagoas, MS, Brasil. Com o doi: http://dx.doi.org/10.23925/2176901X.2017v20i2p461-473 e título: "Reflexões sobre o papel do Bacharel em Gerontologia em Cuidados Paliativos: relato de experiência de uma disciplina", este artigo tem o objetivo de apresentar um panorama sobre os $\mathrm{CP}$ e a relação com a formação do Bacharel em Gerontologia, além de descrever como foi o desenvolvimento de disciplina sobre o tema, e refletir sobre possibilidades de atuação desse profissional nos diferentes níveis de gestão em CP. O gerontólogo pode ter um papel abrangente nos $\mathrm{CP}$, atuando na gestão e avaliação de políticas públicas, nos serviços especializados ou não, e diretamente com o paciente, cuidadores e rede de suporte social, sempre em conjunto com a equipe multiprofissional.

Finalizando este Editorial, os agradecimentos vão, mais uma vez, pelo auxílio recebido do MCTI/CNPq/MEC/CAPES e da PUC-SP, por meio da Pró-Reitoria de Pós-Graduação, por meio do Plano de Incentivo à Pesquisa (PIPEq) / Publicação de Periódicos (PubPer-PUCSP), 2015, para a edição deste periódico. Aos pareceristas do Conselho Científico de nossa Kairós - Gerontologia especialmente, devem-se os mais sinceros agradecimentos por tornarem possível o aperfeiçoamento da escrita científica dos trabalhos aqui inclusos. A mais de uma dezena de pareceristas ad hoc, indicados pelos próprios assessores do Conselho Editorial regular de nossa revista para este número, os maiores agradecimentos por sua boa vontade, presteza e colaboração valiosa com esta Editoria. Por fim, com a palavra os autores, com esta Editoria desejando boa leitura a todos, e se colocando à disposição para o que for necessário nos endereços a seguir:

Flamínia M.M.Lodovici

flalodo@terra.com.br; flodovici@ pucsp.br
Elisabeth Frohlich Mercadante elisabethmercadante@yahoo.com.br

(Editoras Científicas da Revista Kairós Gerontologia)

kairos@ @ucsp.br / http://revistas.pucsp.br/index.php/kairos 